\title{
Semantic congruity and expectancy as separate processes
}

\author{
WILLIAM P. BANKS \\ Pomona College and Claremont Graduate School, Claremont, California \\ and \\ HEDY WHITE \\ Western Carolina University, Cullowhee, North Carolina
}

\begin{abstract}
In two experiments, we investigated the role of expectancy in producing congruity effects in comparative judgment. In Experiment 1, instructions to choose the larger or smaller term either preceded pairs for comparative judgment or preceded individual words for lexical decision. If expectancy in interpreting the comparative judgment terms accounts for the congruity effect, the lexical decision task also should show a congruity effect. However, there were large congruity effects in comparative judgment but not in lexical decision. In this experiment, we used an infiniteset design to make sure that semantic information was needed on comparative judgment trials. In Experiment 2, comparative judgment pairs were preceded by a prime word that either was or was not a category label for the terms in the pairs. There were both congruity and priming effects, with no interaction between the two. This result implies that expectancy and the semanticcongruity effect come from separate processes.
\end{abstract}

In this study, we investigated the relation between semantic priming (or other forms of expectancy, such as perceptual "set") and the semantic-congruity effect. These two effects are found in different experimental paradigms, but they share some fundamental similarities, and it is a matter of no small interest to determine whether they simply represent different versions of the same phenomenon.

Semantic priming is the facilitation of processing of a word after a related word has been registered or responded to. Priming was first identified in an experiment by Meyer and Schvaneveldt (1971). Subjects were shown pairs of letters strings in a lexical decision task, in which they were to decide whether the letters spelled English words. They responded "yes" if both strings spelled words and "no" if at least one string was a nonword. Priming was shown when subjects were faster at correctly classifying an associated pair of words than a pair that was not associated (e.g., they were faster at responding positively to DOCTOR-NURSE than to BUTTER-NURSE). It is as though the associative connection from the first of the two words to be identified facilitated access to the second word in the related pairs. Subsequent research usually has presented the words sequentially, so that the priming and primed word can be identified. Neely (1977) used this presentation technique to show that priming has two com-

This research was supported by NSF Grant BNS 78-17442 and NIMH Grant MN33279 to the first author, and by USPHS Training Grant MH15795 to the second author while she was a postdoctoral fellow at UCLA. Reprint requests should be sent to W. P. Banks, Psychology Department, Pomona College, Claremont, CA 91711 ponents: a fast-acting, automatic facilitation of processing for the primed word that occurs within about $250 \mathrm{msec}$ of the presentation of the prime, and a slower, apparently strategic sort of priming that takes 1-2 sec from the presentation of the prime and seems to depend on subjects' expectations about what will be presented.

The semantic-congruity effect typically is found in the comparative judgment paradigm, which structurally resembles a priming experiment: Subjects first are presented a set of instructions, such as "Choose the larger one," and then are shown a pair of terms, such as HORSE-ELEPHANT. Reaction time for making the choice is measured. The instructions and the pair of stimuli occupy the places of the "prime" and the "primed," respectively. The congruity effect emerges in an interaction between the marking of the comparative adjective ("Choose larger" vs. "Choose smaller") and the end of the magnitude scale from which the stimulus terms are drawn (HORSE-ELEPHANT vs. ANT-FLEA). In this interaction, reaction times (RTs) for comparisons are shorter when instruction form and scale end are congruent (i.e., both are marked or both unmarked) than when they are incongruent. Thus, for example, subjects might be presented with pairs of terms from either the large or the small end of the size scale, and on half of the trials they would be asked to pick the larger, and on half, the smaller of the two. The congruity interaction in this example would occur when "Choose larger" instructions are executed faster than "Choose smaller" for pairs like ELEPHANT-HORSE and when "Choose smaller" instructions are executed faster than "Choose larger" for pairs like FLEA-ANT. 
The similarity between semantic priming and the semantic-congruity effect is evident in the results as well as in the structure of the paradigm. If we assume that congruent combinations of the instructions and stimuli are more closely associated than incongruent ones, then the congruity interaction is, on the face of it, essentially the same as a priming effect. Researchers have, in fact, interpreted the congruity effect precisely as a priming effect (Marschark, 1983; Marschark \& Paivio, 1979, 1981). The mechanism for creating the congruity effect in these models is more generically termed "expectancy," of which semantic priming is but one type. The only other type of expectancy that has been proposed is the perceptual "set" interpretation of the congruity effect (Duncan \& McFarland, 1980).

According to the "set" hypothesis, congruent instructions facilitate the perceptual analysis of the stimuli. Priming, on the other hand, facilitates the semantic interpretation of the terms. In a typical priming experiment (and in the studies reported here), "set" effects and semantic priming would give the same pattern of results. Other operations would be needed to discriminate them.

One strategy previously used to test the expectancy hypothesis should be mentioned before we describe the present approach. This earlier strategy rests on the obvious prediction of an expectancy hypothesis: that the congruity effect should be eliminated if the comparative adjective is presented after the stimulus terms and therefore arrives too late to "prime" them. This prediction has been tested in a number of studies. Both Banks and Flora (1977) and Marschark and Paivio (1979) found only small reductions in congruity effcts when stimuli preceded instructions. These small reductions seem to be attributable to a preprocessing strategy in which subjects prepare the appropriate response while waiting for the instructions to be presented (Banks \& Flora, 1977; Banks, White, Sturgill, \& Mermelstein, 1983). When such a strategy is prevented, congruity effects found with the stimuli first are not smaller than congruity effects found with the instruction first (Banks et al., 1983; cf. also Holyoak \& Mah, 1981).

Although the finding of a congruity effect with stimuli presented before instructions would seem to be decisive evidence against expectancy, it is possible that expectancy still operates, but with the stimulus pair being the prime for the instructions. The congruity effect would then emerge from priming of the instructional term by the stimulus pair (see Marschark, 1983, concerning this argument). This possibility seems quite implausible to us, because congruity effects are often virtually identical when either instructions or stimuli are presented first, but the strength of association between stimuli and instructions typically is not symmetrical. (See Banks et al., 1983, for more discussion of this hypothesis and other evidence against this hypothesis.) Despite the strength of this argument, a crucial test of the hypothesis still is needed. In considering whether to pursue this test, we concluded that the instructions-first versus stimuli-first strategy of attack on expectancy is so general that it affords too many openings for alternative explanations. Further research on effects in this paradigm seemed likely to lead us far away from the questions of interest.

In the present research, we used a test of the expectancy hypothesis that is based on an experiment reported in Banks et al. (1983). In Banks et al.'s experiment, expectancy or semantic priming for the stimulus terms was measured by presenting, on some proportion of trials, a single word (or nonword) after the instructions, instead of the pair of terms to be compared. On the trials having a single string of letters, subjects made a lexical decision rather than a comparative judgment. The single words were drawn either from the repeated list of four words used in the comparative judgments or from a separate list of words naming either large or small things drawn from an "infinite" set (i.e., sampled without replacement). If the instructions facilitated the identification of congruent terms in the way proposed by the expectancy hypothesis, then when single stimulus terms from the list used in comparative judgments are presented for lexical decisions, the ones congruent with the instructions should lead to faster decisions than should the incongruent ones. General preparation for large or small things was assessed by performance on the infinite-set words. The experiment also varied the time interval between onset of comparative adjective and onset of stimulus (this interval being termed stimulus onset asynchrony, or SOA), in order to test for both kinds of priming (automatic and strategic) proposed by Neely (1977).

The results gave good evidence for both of Neely's categories of priming on lexical decision trials but, at the same time, showed the congruity effect to be independent of them. In the lexical decision task, priming operated for the infinite-set words only at the short SOA, and priming operated for words drawn from the list used for comparative judgments only at the long SOA. However, the comparative judgments gave a congruity effect at both SOAs. Banks et al. (1983) concluded that some process other than semantic priming must be responsible for the congruity effect in comparative judgment, since the effect did not covary with either type of priming. They argued that only one available model, the semantic-coding model (see Banks, 1977), could explain the effect without predicting a priming effect.

In the first experiment reported here, we compared priming and congruity effects by mixing lexical decision and comparative judgment trials as in the previous research. However, the experiment differed in an important way: the comparative judgments here were not about a small, repeated set of words. Instead, all of the wordsboth for comparative judgments and for lexical decisionwere drawn from an effectively infinite set. This difference is important because of the necessity of showing that our previous results did not depend on strategies specific to a fixed-list paradigm. If they did, our conclusions would not 
apply to processing of concepts in general, but only to listprocessing situations, hardly the basis of interest in either semantic priming or the semantic-congruity effect.

Over and above our general concern about the use of repeated sets, we note a specific alternative hypothesis that can be eliminated with an infinite-set paradigm. This hypothesis is that subjects may have used a strategy of retrieving the list of terms on each trial after the question for comparative judgment was presented. If they began their retrieval with the items falling at the end of the list specified in the instructions, processing of congruent terms would be speeded relative to processing of incongruent terms and thereby would create a congruity effect in the speed of executing the comparative judgment. The strategy of searching an already retrieved list from the end congruent with the instructions would also create a congruity effect (see Woocher, Glass, \& Holyoak, 1978). We previously have shown that such a list-processing strategy cannot provide a general account of the semanticcongruity effect (see Banks, White, \& Mermelstein, 1980 ), but we have no internal evidence that it was not used in the Banks et al. (1983) experiment in which priming and semantic congruity were unrelated. The possibility of a list-processing strategy in this case vitiates our use of the lack of a relation between congruity and priming to argue against expectancy models. If the congruity effect in this experiment had happened to come from a retrieval or search strategy, then the results would not bear on the applicability of expectancy in the other paradigms. Furthermore, the support for the semantic-coding model from this experiment came from the lack of an alternative that did not predict a relation between priming and congruity. The retrieval or search strategy provides such an alternative and therefore weakens this argument.

Experiment 1 used an infinite-set paradigm and therefore eliminated the possibility of a list-processing strategy. The experiment also varied SOA between presentation of instructions and stimuli for both comparative judgment and lexical decision tasks, in order to determine whether SOA affects the two tasks in the same way. If both effects increased or decreased with SOA, they might rely on a common mechanism, but if they did not, a common mechanism would be rejected. Experiment 2 was conducted because Experiment 1 showed very small (and unreliable) priming effects, and it seemed necessary to show that priming could be demonstrated in lexical decisions in this paradigm. In addition, Experiment 2 found the priming effect (which was reliable) to combine with the semantic-congruity effect additively, an outcome that suggests that the two effects come from independent psychological processes.

\section{EXPERIMENT 1}

\author{
Method \\ Subjects. The subjects were 24 male students from the Univer- \\ sity of California, Los Angeles, who volunteered in order to fulfill \\ partial requirements for a course in introductory psychology.
}

Procedure. Subjects first read instructions explaining that, before each trial, the word "Bigger?" or "Smaller?" would appear on the CRT (a black-and-white Amdek monitor driven by an Apple II+ computer), followed either by a pair of words or by a single letter string that might or might not form a word. If a pair of words followed the instructions, subjects were to press the key to indicate the side of the CRT on which the word designating the bigger or smaller object appeared. If a single letter string appeared, subjects were to press the left key if the letter string formed a word and the right key if it did not. Subjects were instnucted that accuracy and speed were both important but that accuracy was more important.

Subjects received three blocks of 24 trials. The first, a practice block, used stimulus pairs and letter strings that were not used in experimental trials. Order of presentation of the 24 trials in each block was randomized by the computer for each subject. Subjects began a block by pressing either of their response keys. Immediately, instructions to choose either the bigger or smaller term appeared on the screen. Time from onset of instructions to onset of stimulus (SOA) was $650 \mathrm{msec}$ in the practice block, $250 \mathrm{msec}$ in one experimental block, and 1,000 msec in the other experimental block. Order of receiving the two experimental SOAs was counterbalanced over subjects. The stimulus pair or letter string remained in view until the subject responded by pressing the left or right microswitch (as described above). The subject's response caused the computer to erase the stimulus, present feedback ("correct" or "incorrect"), and begin a new instruction-stimulus sequence. The computer recorded RTs for correct responses but not for errors. Because of the infinite-set design, stimuli that led to errors were not repeated.

Design and materials. The 24 trials in each block consisted of eight comparative judgments and 16 lexical decisions. Half of the lexical-decision trials were words and half were nonwords (formed by making one-letter alterations of words, as in Neely, 1977, and in Banks et al., 1983). For both lexical decisions and comparative judgments, half of the trials used instructions to choose the larger term and half used instructions to choose the smaller term, and half of the words designated things from the large end of the size scale and half designated things from the small end of the scale.

The design yielded 48 experimental trials for each subject, composed of the following orthogonal conditions: 2 SOAs $(250 \mathrm{msec}$ vs. $1,000 \mathrm{msec}$ ) $\times 3$ tasks (comparative judgment, lexical decisionword, or lexical decision-nonword) $\times 2$ instructions (choose the larger vs. the smaller term) $\times 2$ size ranges for stimulus words (large vs. small end of scale) $\times 2$ replications of each condition, with a correct response on the left versus right side for the comparative judgment trials

Because of the infinite-set design, in which no subject saw a term more than once, a somewhat complicated between-subject counterbalancing arrangement was required. The instructions and terms for comparative judgment are shown in Table 1 (with size norms from Paivio, 1975, in parentheses). These were arranged into six counterbalancing sets. The counterbalancing ensured that every word was tested in both comparative judgment and lexical decision tasks and was paired with both "Choose larger" and "Choose smaller" instructions. Furthermore, the order of the words in comparative judgment was completely counterbalanced over subjects. The SOA was a between-block variable, and order of administering the two blocks was counterbalanced over subjects. The combination of SOAs and SOA orders with the six counterbalancing sets created 24 counterbalancing conditions, each containing a combination of the experimental variables. One subject was assigned to each counterbalancing condition.

\section{Results and Discussion}

Figure 1 shows congruity effects in RT (means of correct responses) for comparative judgment and lexical decision trials at the 250 - and $1,000-\mathrm{msec}$ SOAs. Note first 
Table 1

Comparative Judgment Pairs Used in Experiment 1 STIMULUS PAIRS

\begin{tabular}{ll}
\multicolumn{2}{c}{ STIMULUS PAIRS } \\
\hline \multicolumn{1}{c}{ Small Things } & \multicolumn{1}{c}{ Big Things } \\
FLEA (1.02)-CHERRY (1.33) & COW (6.47)-ICEBERG (8.80) \\
CRAB (1.98)-PEA (1.22) & CAMEL (7.43)-BOAT (7.10) \\
ANT (1.04)-THIMBLE (1.35) & ELK (6.22)-AIRPLANE (8.73) \\
MOUSE (1.98)-RAISIN (1.27) & BEAR (7.37)-PIANO (6.88) \\
BUTTERFY (1.35)-CRUMB (1.12) & WHALE (8.55)-CAR (7.41) \\
ROACH (1.20)-DIME (1.35) & ZEBRA (6.22)-BUS (8.35) \\
CATERPILLAR (1.53)-PEANUT (1.22) & GIRAFFE (7.69)-TRACTOR (7.20) \\
SNAIL (1.20)-PENNY (1.43) & LION (6.16)-TRUCK (8.12) \\
\hline
\end{tabular}

Note-Size ratings from Paivio, 1975, in parentheses.

that comparative judgment trials (left panels) resulted in strong congruity effects under both SOAs. Congruity effects, as measured by the mean number of milliseconds that each of the four points in the congruity interaction would have to be moved to produce parallel lines, were $82 \mathrm{msec}$ with the short SOA and $135 \mathrm{msec}$ with the long SOA. On the other hand, the expectancy (priming) effect, measured in the same way as congruity effects, at the 250-msec SOA was only $7 \mathrm{msec}$, and it dropped to a reverse congruity effect of $-31 \mathrm{msec}$ at the 1,000 -msec SOA. (Negative effects occur when incongruent instruction-word combinations produce shorter RTs than congruent combinations.) Thus, there were large congruity effects in comparative judgment but virtually no priming effects in lexical decision. Furthermore, SOA did not affect congruity and priming effects in the same way: congruity effects were greater at the long SOA than at the short one, but priming effects were greater at the short SOA than at the long one.

Congruity effects in comparative judgment were similar to those obtained in the repeated-set task in Banks et al. (1983) (66 msec and $95 \mathrm{msec}$ at the 250- and 1,000-msec SOAs, respectively). While Banks et al. (1983) did not use an infinite-set comparative judgment task, they did include an infinite-set lexical decision task, which produced priming effects of $44 \mathrm{msec}$ at the $250-\mathrm{msec}$ SOA condition and $-38 \mathrm{msec}$ at the 1,000 -msec SOA condition. The pattern was similar to the present one, although the magnitude of the priming effect at the short SOA was greater in Banks et al.

In the overall analysis of variance (SOA $\times$ task $\times$ instructions $\times$ big vs. small term), the only reliable main effect was of task $[F(1,23)=78.36, p<.001]$, indicating that lexical decision trials produced shorter RTs (mean $=1,326 \mathrm{msec}$ ) than did comparative judgment trials (mean $=2,184 \mathrm{msec}$ ). The task $\times$ instruction $\times$ scale interaction was reliable $[F(1,23)=9.02, p<.01]$. As Figure 1 suggests, this interaction arises because of the predicted differences between congruity effects in comparative judgment and in lexical decision. Separate $F$ tests confirm that the congruity effect was reliable in comparative judgment $[\mathrm{F}(1,23)=14.31, \mathrm{p}<.01]$, but the priming effect in lexical decision was not reliable $[\mathrm{F}<1.00]$.
As in Banks et al. (1983), we did not find a main effect of SOA (mean RTs were 1,757 msec and $1,753 \mathrm{msec}$ in the 250- and 1,000-msec SOA conditions, respectively), and SOA did not interact with any other variables in the design. Separate F tests indicated that neither the 53-msec difference in congruity effects in comparative judgment under the two SOAs nor the 38-msec difference in priming effects in lexical decision under the two SOAs was

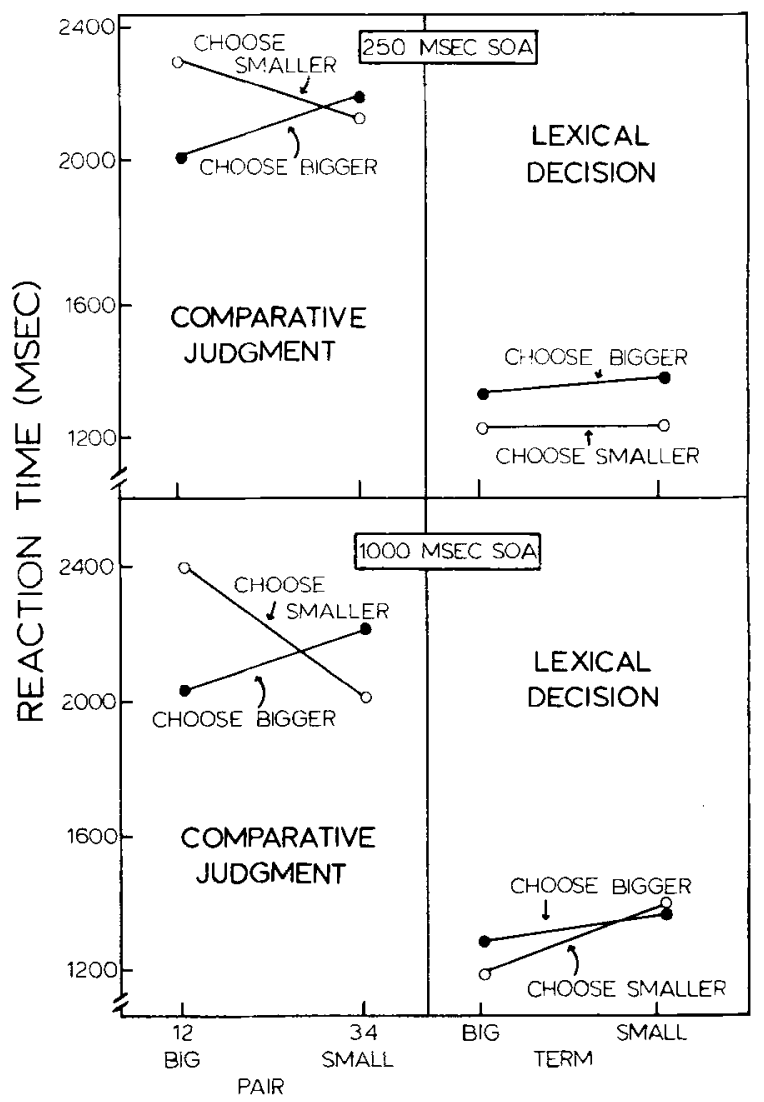

Figure 1. Mean RTs for correct responses (vertical axis) are plotted against the scale end from which terms were drawn (horizontal axis) for instructions to choose terms representing the larger (closed circles) and smaller (open circles) items. The figure shows congruity effects in comparative judgments but not in lexical decisions. In fact, at the 1,000-msec SOA (bottom-right panel), lexical decisions for incongruent instruction-word combinations were faster than decisions for congruent ones. 
reliable $[F s<1.00]$. The latter difference was reliable in Banks et al.

Overall errors for the 24 subjects in the experiment were $6.5 \%$, and errors correlated positively with RTs (collapsing over subjects, $r=.56$ ). An analysis using errors as the outcome measure for the same design as the RT analysis resulted in no reliable main effects. The instruction $\times$ scale interaction was not reliable (although congruent combinations produced $3.6 \%$ fewer errors than did incongruent ones), and there were not any reliable higher order interactions involving congruity or priming effects. The infinite-set design does not allow repetition of trials that led to errors. Hence, errors pose a potential problem for analysis of only correct RTs. When an error occurred, a subject's RT for an equivalent trial (there were 2 trials in each cell of the design) was substituted. Out of the total of 1,152 trials, we had to make this substitution 67 times. In four more cases, both of the two equivalent conditions produced errors, and in these the grand mean for the task was substituted.

In summary, we found strong congruity effects in comparative judgment and did not find priming effects in lexical decision. Indeed, at the longer SOA we found negative effects in lexical decision. These results show that congruity effects in comparative judgment cannot be attributed to expectancy effects, that is, either to semantic priming mechanisms or to "set" effects occurring at early encoding stages of processing. In addition, the results show that list-search or list-retrieval strategies (or, in fact, any strategies based on processing a repeated list) do not account for the congruity effect. This demonstration also was made in Banks and Flora (1977, Experiment 1) and Banks et al. (1983, Experiment 4). The particular importance of the congruity effect here derives from the fact that it was obtained in a situation in which an expectancy effect was not obtained. The previous finding of a congruity effect unrelated to the priming or expectancy effect (Banks et al., 1983, Experiment 1) was open to the argument that, in that case, the congruity effect was only a list-search effect unrelated to semantic processing. In our experiment, the congruity effect must reflect access or use of semantic memory, and yet it still is separable from priming or any other form of expectancy.

\section{EXPERIMENT 2}

This experiment used a comparative judgment task in which a priming word was presented on each trial to create an expectation for one of two sets of terms. This procedure allows us to compare effects of the two sorts of operation (priming and comparative judgment instructions) to determine whether they operate independently of, interfere with, or facilitate each other. If the congruity effect operates essentially by an expectancy mechanism, we might think that, if expectancy is already created by some operation, there would be less room for additional facilitation or interference from another source, and the size of the congruity effect would decrease, almost as though there were a ceiling or floor effect. Thus, we take the prediction of an expectancy hypothesis to be an interference between congruity and priming. However, for reasons we will not elaborate here, Marschark $(1982,1983)$ predicted the opposite interaction: an increase in the congruity effect for expected stimuli and a decrease for unexpected ones. As it turns out, we do not need to settle the prediction of the expectancy hypothesis because our prediction differs from both possibilities: the prediction of the semantic-coding model (Banks, 1977; Banks et al., 1983) for the combination of priming and the congruity effect is independence. The model attributes the two effects to separate and distinct mental operations, and they should be able to operate without influencing each other. Thus, the model predicts that the congruity interaction will be additive with the priming effect.

This experiment used a repeated-list technique with two separate lists whose terms come from two distinct categories: fruits and animals. Within each list, the terms were ordered in size, and size was the dimension used for the comparative judgment. The experiment blocked comparative judgment instructions rather than varying them from trial to trial, as is the more usual procedure. (Blocking does not greatly affect the results of comparative judgment experiments; see Banks, 1977.) The priming stimulus was the cue to list type, "Fruit" or "Animal." It was presented before the stimulus words on each comparative judgment trial and was varied randomly from trial to trial within block. The cue was not correlated with list type, and subjects were made aware of this fact. The reason we chose not to give the prime any predictive value was that, in the comparative judgment paradigm, the instructions also do not predict the level of the pair of stimuli to be judged. Building in a correlation between cues and stimuli or misinforming the subjects on the matter might create a different basis for expectancy effects. We might, thereby, almost artifactually generate an independence between priming and congruity effects that does not reflect the process of interest.

We varied the SOA between prime and stimulus pair in part because we could not be sure which sort of expectancy effect (automatic or strategic) the prime would generate. As it turned out, both sorts of expectancy were generated, although the expectancy effect was not large at the short SOA. In addition, the variation in SOA allowed us to test for the independence between the congruity effect and both the automatic and the deliberate components of expectancy.

\section{Method}

Subjects. The subjects were 13 females and 7 males, drawn from the same pool used in Experiment 1 and who served for approximately $30 \mathrm{~min}$. None of the subjects had participated in Experiment 1

Procedure. The subjects were tested on timed comparative judgments of the ordinal rank of terms drawn from two lists, each containing four terms representing fruits or animals ordered in size. The orderings (with size norms from Banks \& White, 1982, following the items) were: (1) melon (6.06), pear (5.20), lemon (3.62), 
and cherry (1.93); (2) horse (8.59), deer (7.28), chicken (3.17), and rat (2.97).

Subjects first were shown a copy of the two orderings and asked if they disagreed with the placement of any terms. Two of the 24 subjects thought that pears and lemons were the same size but accepted our ordering. There were no other disagreements. Subjects were told that on each trial the word FRUIT or ANIMAL would appear on the screen and would be followed by a pair of terms drawn from either the fruit ordering or the animal ordering. Subjects were told that the category label was not a clue as to which ordering the pair would be drawn from. The experimenter told the subject whether the instructions were to choose the word representing the larger or smaller item, depending upon block (as described below), and explained how to use the response box, as in the comparative judgment trials of Experiment 1.

Subjects received two practice blocks of 24 RT trials each and four experimental blocks of 48 RT trials each. On one practice block and two experimental blocks, the task was to choose the term representing the larger item, and on one practice block and two experimental blocks, the test was to choose the term representing the smaller item. Order of administering blocks was counterbalanced. Under each form of instruction, one experimental block used a 250-msec SOA and the other experimental block used a 1,000msec SOA, with order of the SOAs counterbalanced over subjects. Practice blocks used a 625 -msec SOA.

In each 48-trial experimental block, half of the pairs were preceded by a prime word which was a category label for the terms in the pair (ANIMAL followed by two animal terms or FRUIT followed by two fruit terms), and half of the pairs were preceded by a prime word which was not a category label for the terms in the pair (FRUIT followed by two animals or ANIMAL followed by two fruits). The design yielded 192 experimental RT trials for each subject: 2 (instructions to choose the larger vs. smaller term) $\times 2$ (250- vs. $1,000-$ msec SOA) $\times 2$ (prime related vs. unrelated to stimulus pair) $\times$ 2 (animal vs. fruit terms) $\times 6$ (unique pairs from each 4-term ordering) $\times 2$ (permutations of each pair). In each 24-trial practice block, one permutation of each experimental pair was used, with the term on the left correct for half the trials and the term on the right correct for the other half. The same Apple II+ microcomputer used in Experiment 1 presented prime words and stimuli. The computer recorded RTs for correct responses and numbers of errors (but not RTs for errors). Pairs to which responses were incorrect were presented later in each block.

\section{Results and Discussion}

As seen in Figure 2, both congruity and list-cuing (or priming) effects were obtained. To simplify reporting of results, we plotted in this figure the means of the congruent and incongruent instruction-stimulus combinations rather than the entire congruity interaction. The left-hand panel plots the end pairs from the series of four used for comparative judgment. By convention, these are termed Pairs 12 and 34, in which 1 refers to the largest and 4 the smallest term of the series of four. Pairs 12 and 34 are separated by one ordinal step. The right-hand panel plots results for pairs separated by two ordinal steps, 13 and 24 . The congruity effect was $90 \mathrm{msec}$ overall for the 1 -step pairs and $50 \mathrm{msec}$ for the 2 -step pairs. The overall congruity effect was reliable, as tested by the pair $x$ instruction interaction $[F(5,95)=25.1, p<.001]$, and all eight congruity effects in Figure 2 far exceed the LSD of $33 \mathrm{msec}$ for $\mathrm{p}<.05$.

The data for related and unrelated cues are plotted separately in each panel of Figure 2 . The overall cuing

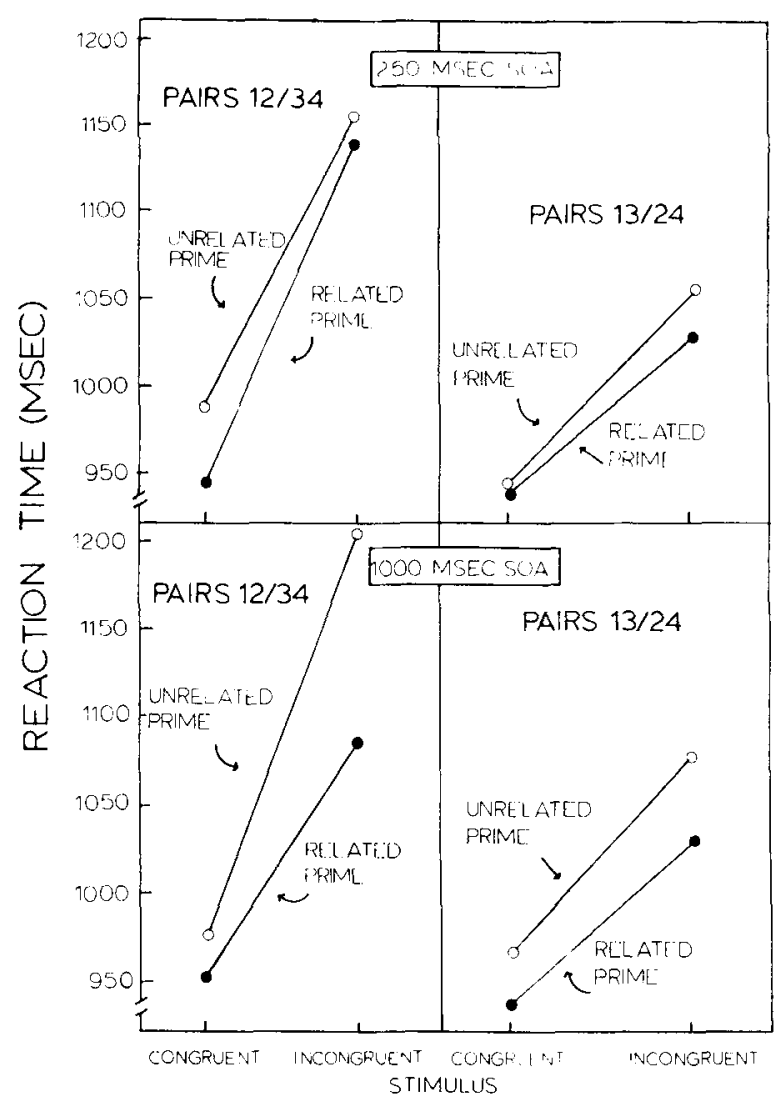

Figure 2. Mean RTs for correct decisions (vertical axis) are plotted against congruence of instruction-pair combinations (horizontal axis) when primes are related (closed circles) and unrelated (open circles) to stimulus pair. The left panels use RTs for Pairs 12 and 34 and the right ones use RTs for Pairs 13 and 24. The figure shows main effects for cuing and congruity but no interaction between them. SOA did not interact with the congruity effect but it did interact with the cuing effect, which was greater at the 1,000 -msec SOA (bottom panel) than at the 250 -msec SOA (top panel).

effect was $21 \mathrm{msec}$ at the $250-\mathrm{msec}$ SOA and $69 \mathrm{msec}$ at the 1,000 -msec SOA. This effect is reliable $[F(1,19)$ $=20.2, \mathrm{p}<.001]$. The difference in cuing between the two SOAs is also reliable $[F(1,19)=4.68, p<.05]$, suggesting that the strategic component of priming here is stronger than the automatic component, even though there was no strategic advantage to using the cue.

Whereas the cuing effect showed a large, reliable increase from an SOA of $250 \mathrm{msec}$ to one of $1,000 \mathrm{msec}$, the congruity effect was virtually constant at 69 and $70 \mathrm{msec}$ at SOAs of 250 and $1,000 \mathrm{msec}$, respectively. Furthermore, the cuing effect had no apparent systematic influence on the congruity effect. For the pairs separated by one step (Pairs 12 and 34), the expected set gave a larger congruity effect than did the unexpected at the 250msec SOA, but the unexpected set gave a larger congruity effect than did the expected at the 1,000-msec SOA. The reverse was the case for the two-step pairs (13 and 24). Overall, the expected set gave a mean congruity effect of $65 \mathrm{msec}$, and the unexpected set gave one at $75 \mathrm{msec}$. None of these differences is reliable, and the $F$ ratios are 
less than 1.0 for all orders of interaction involving congruity and cuing.

The conclusion that expectancy and the congruity effect are additive depends on accepting a null hypothesis, and it is important to be sure that the probability of a Type II error is not high. First, the low $F$ ratio $(F=0.81$, $\mathrm{p}=0.55$ ) seems to come from a small effect, $10 \mathrm{msec}$ per point in the interaction, rather than from a noisy experiment, with MSe $=39$, and an estimated SE of $7.7 \mathrm{msec}$. Second, the interaction shown in Figure 2 is not reliable $(F=1.56, p=0.18)$. This insignificant interaction implies that the apparently superadditive interaction in the lower left-hand corner (Pairs 12/34 at the 1,000-msec SOA) does not represent a reliable deviation from the general lack of interaction. To give more power to this test, we again computed this $\mathrm{F}$ ratio with a pooled error term that included all interactions between subjects and the combinations of variables in this interaction. The resulting $F(5,741)$ was 1.57 , essentially the same as in the unpooled test and still not passing the $p=0.10$ level. Third, the interaction between congruity and expectancy for Pairs $12 / 34$ at the SOA of $1,000 \mathrm{msec}$ (see Figure 2) was $26.25 \mathrm{msec}$ per point, which falls short of the LSD of $30 \mathrm{msec}$ for this interaction.

Mean error rate was $4.1 \%$, with a correlation of .58 between RT and errors over the main variables of the experiment. An ANOVA of the errors had no reliable effects of interest but did show a (nonsignificant) reverse of the congruity effect for both 1-step pairs (minus $2.5 \%$ and 2 -step pairs (minus $1.9 \%$ ). Such a negative congruity effect in the errors raises the concern that the congruity effect in the RTs is contaminated by a speed-accuracy trade-off. None of the interactions between the congruity effect and cuing shows a possible speed-accuracy effect, but nevertheless a trade-off could pose a problem for interpretation.

In order to obtain data free of the potential for a speedaccuracy trade-off, we analyzed each subject's errors in the congruity interaction (collapsing over the 1-step and 2 -step pairs). This analysis revealed that 10 subjects had a positive congruity effect in their error rates and 10 had one that was either negative or exactly zero. We then submitted the RTs of the 10 subjects with positive congruity effects in their errors to the same analyses performed on the full set of data. The results were very similar to those of the full set of data.

\section{GENERAL DISCUSSION}

The results show that priming and the semanticcongruity effect have different functional properties and therefore are likely to result from different psychological processes. Experiment 1 mixed the comparative judgment and lexical decision tasks, using an infinite-set procedure in both cases to ensure that subjects used semantic memory information (rather than a memorized list) in both tasks. The priming effect was small at the 250-msec SOA and decreased to a negative effect at the $1,000-\mathrm{msec}$ SOA, while the congruity effect was large and increased from the short to the long SOA. In Experiment 2, priming took the form of giving noninformative cues for one of two prememorized lists, and comparative judgments were about these same prememorized lists. Both effects (priming and semantic congruity) were therefore assessed in a fixed-list situation and both were reliable. However, the two effects in this experiment were independent of each other in the sense that there was no systematic interference or facilitation between them. They also were independent in that the semantic-congruity effect was constant over SOA, whereas the priming effect increased greatly from the $250-\mathrm{msec}$ to the 1,000 -msec SOA.

One possible argument we can reject is that the semantic-congruity effect is still a priming effect, but the expression of priming is different in comparative judgment and lexical decision. In the one case, there were two words to perceive and decide about, while in the other case, only a single word. Processing two words may greatly increase effects that are small when only one word is processed. This argument would be a good one if we had found merely a difference in size between semantic congruity and priming effects, but we also found a difference in the way these effects vary with SOA. Furthermore, in the present Experiment 1, the priming effect was extremely small at the short SOA, which gave a large congruity effect, and it was negative at the long SOA, which gave a still larger (and positive) congruity effect. It is difficult to see how the small priming effect could be multiplied into the larger congruity effect found at the short SOA. It seems impossible for a negative priming effect to convert into a positive congruity effect.

Another matter we should consider is whether priming, as measured in the lexical decision task, covers all varieties of process that could be termed expectancy. The only expectancy process that has been proposed as an alternative to semantic priming is perceptual "set" (i.e., a facilitation of visual encoding; Duncan \& McFarland, 1981). However, if "set" had been induced here, it would have had the same pattern of facilitation and interference as priming. In fact, the present test for priming in lexical decision does not discriminate between "set," priming, and any other form of readiness for one stimulus as opposed to another. There are operations that could discriminate between "set" and priming, but we did not attempt this discrimination in this article.

One matter of concern is the extent to which the conclusions depend on an implicit additive model. Given the assumption of discrete serial stages, the lack of interaction between congruity and cuing implies that they reflect the operation of different stages. However, if we relax this assumption, what conclusions can we draw? McClelland (1979) has worked out the consequences of a different set of assumptions in some detail. He developed what he termed the "cascade" model, in which each information-processing stage continuously passes information to the following stage, which begins operating on this partial information without waiting for the previous 
stage to be completed. These assumptions differ from those of the semantic-coding model, in which the coding operations await the outcome of perceptual registration, and choice follows coding. If the stages of the semantic coding model are allowed to overlap as in the cascade model, patterns of interaction and additivity do not yield the same inferences about independence of stages.

In the present pattern of data, in which congruity and cuing do not interact, McClelland (1979) has shown that two different theoretical conclusions are possible. One is that the two factors affect the rates of two different processes. This is equivalent to the discrete-stage inference that the two factors affect the durations of different psychological processes, which is the conclusion we drew initially. The other possibility is that congruity and cuing affect the same process, but in different ways. According to McClelland's analysis, if a stage is a relatively fast one and if one factor affects the rate with which that stage processes information and the other affects the same stage's asymptotic level of completion of processing, then the combination of the two factors will be additive in reaction time. We have some preliminary data on speedaccuracy trading functions that suggest that the congruity effect shows up in the rate parameter of the informationactivation function. If so, then the cuing variable must affect only the asymptote for the single-stage inference to hold. However, McClelland presented analyses showing effects of priming on both parameters, therefore suggesting that the single-stage interpretation cannot be correct. We are dubious also about the single-stage interpretation for another reason: the congruity effect seems to take place in a relatively long stage, not a fast one. Congruity effects range into the hundreds of milliseconds and can consume $10 \%-20 \%$ of processing time. The single-stage model gives additive results only when the process is a relatively fast one.

Finally, we should consider what models of the congruity effect are, supported by these results. To begin with, two models are rejected. First, the model proposing search or retrieval of the list for comparative judgment does not explain the congruity effect with an infinite-set list. Second, the expectancy models that attribute the congruity effect to "set" effects or semantic priming effects are rejected. These models cannot explain the fact that the pattern of facilitation and interference is different when the very same words used in the comparative judgment are individually presented for lexical decision. Rejection of these two models implies that the congruity effect is not a result of any sort of expectancy; that is, it does not take place in aspects of the process related to perception, identification, or retrieval of the terms presented for comparative judgment.

We would argue instead that the congruity effect arises from the way memory information about the stimuli is used in the choice process. One model of the choice process is the semantic-coding model (see Banks, 1977; Banks et al., 1983), which ascribes the congruity effect to patterns of match and mismatch between the stimuli as coded and the instructions. If, for example, the stimulus words name two large things, they both would be encoded as large, or in codes that we represent as $\mathrm{L}$ and L+. The instructions "Choose larger,'" encoded as L+, would find an easy match for response selection, but "Choose smaller" ( $S+$ ) would not. To make the proper association between a pair of large things ( $\mathrm{L}$ and $\mathrm{L}+$ ) and the "Choose smaller"' instructions $(\mathrm{S}+)$, or between a pair of small things and "Choose larger" instructions, either the instructions or the stimuli would need to be recoded, and this recoding would add to the RT. Both of the incongruent stimulus-instruction combinations would therefore take longer than would the congruent combinations, producing to the congruity interaction.

Another explanation of the congruity effect, the semantic interference model, was suggested by Banks and Root (1979). They proposed that the effect may come from a sort of Stroop interference between the semantic level of the stimuli and the instructions, rather than from the specific code-matching process of the semantic-coding model. This explanation is not bound to assumptions about processing "stages," but it fails to specify the process underlying the effect. Our main conclusion that expectancy and congruity affect different stages in terms of this explanation would be that congruity and expectancy effects are isolable as separate aspects of performance with meaningful stimuli. Testing this conclusion would require accepting some assumptions, such as, for example, processing stages or explicit mechanisms of interference and facilitation.

In conclusion, whether the semantic-coding model or the Banks and Root (1979) semantic interference model or some other model is ultimately accepted, the present results, combined with previous ones, show that the semantic-congruity effect does not take place as a result of the relative difficulty or ease of perception or retrieval of information used in the task. Instead, the effect results from the relative ease with which different coded representations of the stimuli can lead to the selection of the correct response.

\section{REFERENCES}

BANKS, W. P. (1977). Encoding and processing of symbolic information in comparative judgments. In G. H. Bower (Ed.), The psychology of learming and motivation (Vol. 11, pp. 101-159). New York: Academic Press.

Banks, W. P., \& Flora, J. (1977). Semantic and perceptual processes in symbolic comparisons. Journal of Experimental Psychology: Human Perception \& Performance, 3, 278-290.

Banks, W. P., \& Root, M. (1979). Semantic congruity effects in judgments of loudness. Perception \& Psychophysics, 26, 133-142.

Banks, W. P., White, H., \& Mermelstein, R. (1980). Position effects in comparative judgments of serial order: List structure vs. differential strength. Memory \& Cognition, 8, 623-630.

Banks, W. P., White, H., Sturgill, W., \& Mermelstein, R. (1983). Semantic congruity and expectancy in symbolic judgment. Journal of Experimental Psychology: Human Perception \& Performance, 9 , 560-582.

Duncan, E. M., MCFarland, C. E. (1980). Isolating the effects of symbolic distance and semantic congnuity in comparative judgments: An additive-factors analysis. Memory \& Cognition, 8, 612-622. 
HOLYOAK, K. J., \& MAH, W. A. (1981). Semantic congruity in symbolic comparison: Evidence against an expectancy hypothesis. Memory \& Cognition, 9, 197-204.

MARSChARK, M. (1982). Expectancy, equilibration, and memory. In J. Yuille (Ed.), Imagery, memory and cognition: Essays in tribute to Alan Paivio. Hillsdale, NJ: Erlbaum.

MARSCHARK, M. (1983). Semantic congruity in symbolic comparisons Salience, expectancy, and associative priming. Memory \& Cognition, 11, 192-199.

Marschark, M., \& Paivio, A. (1979). Semantic congruity and lexical marking in symbolic comparisons: An expectancy hypothesis. Memory \& Cognition, 7, 175-184.

Marschark, M., \& Paivio, A. (1981). Congruity and the perceptual comparison task. Journal of Experimental Psychology: Human Perception \& Performance, 7, 290-308.

MCClelLAND, J. L. (1979). On the time relations of mental processes:
An examination of systems of processes in cascade. Psychological Review, 86, 287-330

Meyer, D. E., \& Schvaneveldt, R. W. (1971). Facilitation in recognizing pairs of words: Evidence of a dependence between retrieval operations. Journal of Experimental Pscyhology, 90, 227-239.

Nefly, J. H. (1977). Semantic priming and retrieval from lexical memory: Roles of inhibitionless spreading activation and limitedcapacity attention. Journal of Experimental Psychology: General, 106, 226-254.

Palvio, A. (1975). Perceptual comparisons through the mind's eye. Memory \& Cognition, 3, 635-647.

WoOcher, F. D., Glass, A. L., \& Holyoak, K. J. (1978). Positional discriminability in linear orderings. Memory \& Cognition, 6, 165-173.

(Manuscript received November 6, 1984; revision accepted for publication September 5, 1985.) 